Recepción: 12/ 06/ 2017

Aceptación: 10 / 07/ 2017

Publicación: 15/ 11/2017

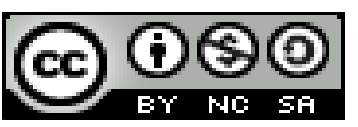

Ciencias de la educación

Artículo de investigación

\title{
La gestión del cambio desde la teoría y la práctica
}

Managing change from theory and practice

Gerenciando mudanças da teoria e da prática

Gerardo V. Villacreses-Álvarez ${ }^{\mathrm{I}}$ gerardo30208@gmail.com

Correspondencia: gerardo30208@gmail.com

I Psicólogo Mención Psicología Industrial Organizacional, Universidad Laica Eloy Alfaro de Manabí, Manta, Ecuador. 


\title{
Resumen
}

El término Change Management se está utilizando en la actualidad en la mayoría de las empresas u organizaciones pues es la forma que existe de que las empresas estén preparadas para el futuro. Este trabajo tiene como objetivo analizar teóricamente lo que significa la gestión del cambio y mostrar una experiencia práctica dentro de una Institución de Educación Superior (IES). Se basa en el análisis documental, fundamentalmente de libros y artículos, donde prevalece el análisis a través de la experiencia y la inducción - deducción. Se tratan aspectos importantes que deberían incluirse en los temas relacionados con la gestión del cambio y se muestra un ejemplo práctico relacionado con la Universidad Laica Eloy Alfaro de Manabí.

Palabras clave: gestión del cambio; instituciones de educación superior; liderazgo, comunicación.

\begin{abstract}
The term Change Management is currently used in most companies or organizations because it is the way that companies are prepared for the future. The objective of this work is to theoretically analyze what change management means and show practical experience within a Higher Education Institution (IES). It is based on the documentary analysis, fundamentally of books and articles, where the analysis prevails through experience and induction - deduction. Important aspects that should be included in the topics related to change management are discussed and a practical example is shown related to the Lay University Eloy Alfaro de Manabí.
\end{abstract}

Keywords: change management; higher education institutions; leadership, communication.

\section{Resumo}

O termo Gerenciamento de Mudanças é atualmente usado na maioria das empresas ou organizações porque é a forma como as empresas estão preparadas para o futuro. O objetivo deste trabalho é analisar teoricamente o que significa gerenciamento de mudanças e mostrar experiência prática dentro de uma Instituição de Ensino Superior (IES). Baseia-se na análise documental, fundamentalmente de livros e artigos, onde a análise prevalece através da experiência e indução - dedução. Os aspectos importantes que devem ser incluídos nos tópicos 
relacionados ao gerenciamento de mudanças são discutidos e um exemplo prático é mostrado em relação à Universidade Leiga Eloy Alfaro de Manabí.

Palavras chave: gerenciamento de mudanças; instituições de ensino superior; liderança, comunicação.

\section{Introducción}

En este mundo globalizado los cambios son frecuentes y no se detienen, lo que obliga a los seres humanos a estar listos y preparados para lo que depara el futuro (condiciones y circunstancias). Sin embargo, existen personas que le tienen miedo al cambio y siguen la monotonía de las mismas actividades todos los días.

La oferta de nuevos servicios y las nuevas fuentes de trabajo van de la mano en un mundo globalizado. Las antiguas tradiciones de vivir y trabajar están cambiando esto es ocasionado por el resultado de la coacción de la sociedad y las empresas.

Robbins (2004), establece que el cambio surge de la necesidad de romper con el equilibrio existente, para transformarlo en otro, mucho más provechoso financieramente hablando, donde se establece qué fuerzas deben romper con el equilibrio, interactuando con otras fuerzas (sociedad y empresas) que tratan de oponerse, (resistencia al cambio). Es por ello que cuando en una organización se plantea un cambio, debe implicar un conjunto de tareas para tratar de minimizar esta interacción de fuerzas.

Cristian Benavente (2011) clasifica en 4 grupos los factores que hacen que una organización sufra cambios:

1.- Cambio del ambiente del mercado

2.- Cambios tecnológicos

3.- Cambios en los requerimientos competitivos

4.- Cambios internos de la empresa

Lo que plantea Benavente (2011), tiene mucha relación con lo que expuesto por Robbins (2004), “-cambiar o morir-es el grito de aliento entre los administradores de todo el mundo" (p556). Este 
autor, señala algunas fuerzas de impulsos de cambio: 1.- La tecnología, 2.- La naturaleza del trabajo, 3.- La crisis económica, 4.- Las tendencias sociales y 5.- La política mundial.

Este trabajo tiene como objetivo analizar teóricamente lo que significa la gestión del cambio y mostrar una experiencia práctica dentro de una Institución de Educación Superior (IES).

\section{Metodología}

Este trabajo se basa en el análisis documental, fundamentalmente de libros y artículos. Además, prevalece el análisis a través de la experiencia y la inducción - deducción.

\section{Desarrollo}

Las organizaciones o empresas tienen que tener una respuesta inmediata, rápida y flexible a los cambios del medio ambiente para existir a largo plazo. Existen empresas que tienen ya un historial de cambios dentro de su institución, tienen ya una metodología y experiencia de cómo y cuándo deben generar un cambio. Así se tiene un mayor tiempo de planificación de cómo realizar un cambio y generar nuevos procesos.

Dentro de una organización, antes de comenzar un cambio, Benavente (2011) muestra que se tienen que realizar varias preguntas fundamentales (dependiendo la naturaleza del negocio) como ejemplo: ¿Hay fluctuaciones de la cifra de ventas?, ¿hay un control de la configuración del precio?, ¿hay el peligro de productos substitutos?, ¿hay una tendencia en la empresa a perder clientes importantes?

Estas preguntas pueden variar por la actividad del negocio, por ejemplo, se puede formular la siguiente pregunta para una Institución de Educación Superior: ¿El modelo de educación actual cubre las demandas y exigencias de las empresas?

El término Change Management también se encuentra muchas veces asociado al término "desarrollo organizacional", sin embargo, el "desarrollo organizacional" solo es una parte del Change Management. (Kraus, Becker-Kolle, \& Fischer, 2004) 
En los años 40 Kurt Lewin realizó algunos experimentos donde uno de ellos fue la teoría del pionero $(1947,1958)$, que está relacionada con cambios en una organización de diferentes fases.

El Change Management tiene sus orígenes en los Estados Unidos, en los años 50 del siglo 20. Los científicos Roethlisberger y Mayo realizaron estudios en relación al aumento de rendimiento en las fábricas de Western Electric (compañía eléctrica), la misma que en el año 1984 fue renombrada AT\&Technologuies.

Hasta los años 60 la mayoría de las organizaciones eran jerárquicas, es decir con un jefe en la cabeza. La organización daba algo por hecho y la autoridad del jefe estaba aceptada en cada caso.

Posteriormente en los años 70 comienza otro factor que generó cambios dentro de las empresas y fue el deseo de los empleados de tener participación en las empresas donde da inicio y surge el 'entrenamiento de equipo', 'desarrollo del equipo'.

Este nuevo enfoque siguió dentro de los siguientes años, lo que llevó que en los años 80 se empiece a hablar de 'trabajo en equipo' y se convirtiera en lo que conocemos como desarrollo organizacional.

Cuando se analiza el artículo de Benavente (2011), se muestra que el Change Management confronta de forma directa con todos los retos de un ambiente que se transforma continuamente y resulta una orientación nueva, a lo que se puede definir como una visión estratégica que puede ir desde la gerencia hasta sus canales de comunicación, de esta teoría nacen preguntas como ¿Qué tiene que cambiar la organización? ¿Cómo tiene que cambiar? ¿Cómo puede garantizar que todo funciona sustentablemente?

Change Management posee 4 pasos fundamentales que son: 1.- El análisis del problema, 2.- La planificación (determinación de las metas, elección de los conceptos de cambio,), 3.Transformación de los cambios (aprender de los nuevos comportamientos, integración y motivación de los trabajadores, perfeccionamiento), 4.- Control del éxito (seguridad de éxito, modificaciones). 
Pero hoy existe resistencia al Change Management, que se da cuando el empleador no es capaz ni está dispuesto a transformar y compartir cambios en la empresa. El Change Management tiene como meta principal el modelar el cambio organizado y a causa de eso implica en cada empresa un factor de éxito.

Uno de los factores que hace que el Change Management fracase en algunas empresas es la resistencia de los empleados, también la lucha de poder en las organizaciones.

La naturaleza del hombre tiende a afectarse por los cambios, solo algunas personas ven el cambio como una oportunidad de crecimiento y mejoramiento, lo que conlleva que en algunas organizaciones fracase el cambio. El ser humano reacciona a cambios por su naturaleza y lo hace de forma negativa cuando lo afecta personalmente. Muchas veces los cambios pueden ser sorprendentes y en otros incómodos que conllevan a que el empleador o trabajador tenga que desligarse de algunas costumbres.

Los científicos definen la resistencia, como un tratamiento para salvar un status-quo, esto que quiere decir que los trabajadores y empleadores no quieren salir de su zona de confort laboral y no experimentar nuevos procesos. Es ahí donde se tiene que explicar de manera detallada en qué consisten los cambios que se plantean, ya que uno de los factores de negativa para un cambio es el falso entendido del problema, porque están pensando que todo funciona bien. Otra razón es el miedo a lo desconocido.

Otra razón muy importante es no tener buenos canales de comunicación (comunicación deficiente). Esto se da debido a que los empleados reciben informaciones insuficientes sobre el cambio que la organización o empresa va a tener. Sobre todo, en muchos casos los empleados no tienen confianza en la gerencia. Otro factor es que los empleados tienen miedo a realizar trabajos extras.

Analizando el contexto del cambio organizacional se puede plantear algunas rutas para que el cambio sea favorable y acogido en la empresa. Durante la planificación del cambio es aconsejable incluir las posibles resistencias. Con este método se pueden disminuir problemas adicionales, que se surgen de resistencias no contempladas. Es importante, en primer lugar, ganar 
a los potenciales compañeros de lucha, después la organización tiene que animar los compañeros de lucha críticos y al final tiene que ser capaz de persuadir a los incorregibles.

Existen distintas teorías del cambio institucional. Una de ellas es 'la erosión o discontinuidad de una práctica o actividad previamente institucionalizada'.

Otros autores, apuntan a que los cambios ocupan un espacio de suma importancia en la actualidad, como lo indica Vargas (2000) cuando manifiesta que los impulsadores de los cambios organizacionales en esta época post moderna tienen influencia en el mundo en la parte económica, social y política y, por lo tanto, se ve afectado el mundo de las organizaciones de diferentes formas por lo que pone a pensar formas y estilos de vida acorde a las nuevas demandas de la época.

La evolución de los enfoques teóricos en sus administraciones; particularmente en la provincia de Manabí con el surgimiento de las primeras empresas procesadoras de pescado, se aplican una variedad de modelos organizativos de diferentes naturalezas, pero todas ellas desde la empresa más antiguas o con más personal, hasta las más nuevas buscan una mayor producción a menor costo y poco o nada importa el ser humano como tal, este es un símil que se vive en las instituciones públicas también, si vemos que en la mayor parte de la historia del Ecuador, se ha vivido bajo un modelo de desarrollo sostenido en el capital. Amerita la necesidad de mejorar y sostener la producción o rendimiento, pero desde un análisis de la importancia del principal motor de la empresa o institución: el humano.

"El cambio surge de la necesidad de romper con el equilibrio existente, para transformarlo en otro, mucho más provechoso financieramente" (Robbins, 2004). Donde las fuerzas deben romper con el equilibrio interactuando con otras fuerzas que tratan de oponerse, (resistencia al cambio) es por ello que cuando en una organización se plantea un cambio, debe implicar un conjunto de tareas para tratar de minimizar esta interacción de fuerzas, es ahí donde se pone de manifiesto el Liderazgo Transformacional (Bass \& Avolio, 1990), que defiende la existencia de dos tipos de liderazgo: el transaccional y el transformacional, este último considerado el más importante, y sobre el que habría que desarrollar propuestas que motiven su desarrollo. 
En vista de lo mencionado por Robbins (2004), decimos que existen varios factores que interfieren en el cambio organizacional, que en muchos casos son ajenos a la voluntad de los trabajadores por ser factores externos y no internos. Un ejemplo claro son las políticas de cada país que afectan de forma favorable o negativa la producción de las organizaciones. La inadecuada aplicación de este factor externo puede ser dañino para el crecimiento industrial de las empresas, como se ha podido observar en varias ocasiones con fábricas, que aplican leyes de contratos a través de la figura de tercerizaciones.

En el Ecuador, se toma como ejemplo de factores que interfieren en las organizaciones, cuando las empresas empacadoras y procesadoras de pescado en la provincia de Manabí se ven afectadas por el bajo costo de sus productos y la escases de los productos del mar (naturaleza del negocio), cuyas secuelas se va en desmedro de los empleados y trabajadores, con políticas de recorte, inestabilidad laboral, mayor presión al trabajador, entre otros, que ocasiona una inseguridad laboral en el empleado o trabajador.

En la época actual, que se apuesta en el país, por rescatar el valor humano sobre el capital, defendiendo los derechos del trabajador sobre el valor monetario, se debe enfocar lo señalado por Robbins (2004), más específicamente en las tendencias sociales y políticas mundiales que son desde nuestro punto de vista como Psicólogo Organizacional las principales responsables que las empresas busquen un cambio en sus organizaciones por falta de productividad (ganancias), y donde se considera que para lograr un cambio a favor de una mejor productividad, basada en el ser humano, se tiene que enfocar en un Liderazgo Transformacional, pues es una herramienta fundamental para desarrollar un cambio organizacional y mejorar la producción en los trabajadores de las empresas.

Durante las dos últimas décadas, el Cuestionario de Liderazgo Multifactor (MLQ) ha sido desarrollado y validado (Avolio, Bass, \& Jung, 1999). Ahora es el instrumento estándar para evaluar una gama de actividades de transformación organizacional.

La eficacia del liderazgo transformacional ha sido probada en muchos países. El instrumento se administra a los subordinados en una serie de muestras heterogéneas en Alemania. Los resultados demuestran alta validez factorial y convergente, así como la consistencia interna de las 
organizaciones. En resumen, el MLQ con sus nueve escalas de liderazgo y tres escalas de resultados son ahora aplicables en un sinnúmero de organizaciones.

Sería útil aplicar este instrumento en la universidad y diagnosticar la tipología de liderazgo que permita desarrollar un plan de intervención para mejorar el nivel de compromiso del personal que labora, frente a la misión institucional.

Ahora bien, no puede haber una cultura organizacional de éxito, sino hay una adecuada salud ocupacional de los trabajadores que refleje la satisfacción del cumplimiento de sus labores. Un eje fundamental de toda intervención organizacional debe ser la transversalización de políticas de seguridad laboral en cada una de las etapas de cambio de entorno laboral que se quiera desarrollar.

La seguridad y salud en el trabajo es un campo interdisciplinario que engloba la prevención de riesgos laborales inherentes a cada actividad. Su objetivo principal es la promoción y el mantenimiento del más alto grado de seguridad y salud en el trabajo. Esto implica crear las condiciones adecuadas para evitar que se produzcan accidentes de trabajo y enfermedades profesionales.

Vargas (2000), expresa que los acelerados cambios que se están efectuando en estos tiempos de posmodernidad, afectan "todos los órdenes, económicos, sociales y políticos y, por lo tanto, también afectan el mundo de las organizaciones. Por supuesto, todos estos cambios están modificando maneras de pensar, formas y estilos de vida, quehaceres y actividades de los individuos". (Vargas, 2000)

Oria (2002), opina que en estos tiempos la acción humana directa se ha simplificado es decir que la introducción de las nuevas Tecnologías de Información y Comunicación en las organizaciones, ha llevado a simplificar la acción humana, además esta autora también señala que:

"La acción humana se ha simplificado, el tiempo de ejecución se ha reducido sensiblemente, la tecnología encierra un importante volumen de conocimiento acumulado y una buena parte del proceso escapa a nuestro control directo, se encuentra bajo el control de sistemas expertos, cuyas lógicas podemos llegar a comprender, pero la posibilidad de modificar o intervenir en las 
mismas, no se encuentra accesible al usuario común. Nuestras acciones cotidianas se han transformado, son más simples y más complejas a la vez, en la medida que lo analicemos desde el ángulo de las intervenciones que habilitan los procesos pautados fuertemente por la tecnología”. (Oria, 2002)

Otros autores que han estudiado el cambio organizacional, (Maldonado \& Pérez, 2006), coinciden con lo que plantea Robbins (2004) al exponer la necesidad de que las prácticas laborales estén dirigidas a crear un clima o atmósfera afectiva.

Analizando las diferentes teorías de los distintos autores y llevándolo al contexto de la Universidad Laica Eloy Alfaro de Manabí que está ubicada en la ciudad de Manta, se puede decir que la educación superior está en cambios. Esta IES, que al inicio el CEAACES (Concejo de Evaluación, Acreditación y Aseguramiento de la Educación Superior) la ubicó en la categoría D considerada universidad no acreditada en el periodo del anterior Rector, atravesó el cambio organizacional para poder acreditarse y más aún esta IES es considerada la tercera más grande del país e institución símbolo de Manabí. El cambio organizacional comienza cuando el CES (Concejo de Educación Superior) decide en el año 2015 la Intervención Integral de esta Universidad, que al inicio tuvo mucha resistencia desde los empleados, por desconocimiento y por falta de canales de comunicación.

La Universidad Laica Eloy Alfaro de Manabí, pasó de una dinámica organizacional, liderada por un único rector que dirigió la institución por más de 25 años, a un nuevo estilo de liderazgo que ha marcado las dinámicas internas, que se han visto reflejados en cambios estructurales, que desdibujan nuevos problemas organizacionales.

Las instituciones públicas, como es el caso de las universidades también tienen un sistema organizacional, que muchas veces se ve afectado por la aplicación de débiles políticas de desarrollo, y sobre todo por la aplicación de leyes de evaluación para acreditación, -que nacieron desde hace pocos años-, que ocasionaron el surgimiento de nuevos procesos, y generaron cambios en las organizaciones y en las dinámicas internas del sistema de universidades. 
Es así que esta IES pasó de tener un Rector que estuvo en el poder por más de 25 años con autoridades ya adueñadas de los puestos, donde tener ideas de mejorar los procesos era sinónimo de estar en contra de la autoridad-, a una universidad que en la actualidad ya está acreditada a solo 4 puntos de la categoría B. Todo esto se da por el cambio organizacional y el empoderamiento institucional de los trabajadores, mejorando procesos que llevan a tener mejores resultados, donde los decanos, directores jefes de área son medidos por su desempeño y ya no son dueños de los puestos como era antes. Esta Universidad es el claro ejemplo de un cambio organizacional aun cuando es más difícil buscar un cambio en una institución pública que en las privadas, ya que en las primeras se depende del ejecutivo y más aún del factor político, y en el segundo se depende de los dueños y su capacidad de buscar el éxito.

En la actualidad la Universidad Laica Eloy Alfaro de Manabí cuenta con un Rector que ganó las elecciones en febrero del 2016, al cual se debe el nuevo modelo que esta IES tiene y la nueva visión empezando con un PEDI (Plan Estratégico de Desarrollo Institucional) donde se enmarcan los nuevos objetivos de la ULEAM.

La aplicación del Change Management en las organizaciones o empresas depende de un factor muy importante que es el liderazgo que los jefes o gerentes tengan en la organización. Se pueden tener todos los factores que coadyuven a tener un cambio favorable, sin embargo, todos estos factores pueden ser insuficientes ante la falta de liderazgo de los jefes o gerentes. Esto quiere decir que hay que comenzar el cambio con la consulta a sí mismos de los gerentes o jefes para ver si están dispuestos a cambiar o ser entrenados para encontrar el éxito en la organización, ¿pero qué liderazgo es el adecuado?, ¿el transaccional o el transformacional? Este último es el considerado el más importante, y sobre el que habría que desarrollar propuestas que motiven su desarrollo.

Ante lo mencionado se puede decir que el liderazgo transformacional es la clave para que el cambio organizacional tenga el efecto y el respaldo necesario en las organizaciones. Cuando se tiene un líder que empodera a los trabajadores, donde los canales de comunicación son fluidos, se suprimirán los factores de resistencia al cambio, es en esta nueva corriente del liderazgo en la que hay que enfocarse. Y en el artículo de Cristian Benavente (2011) se hace hincapié en los pasos 
para obtener un cambio organizacional y sus estructuras, sin embargo, no hace referencia a la importancia un liderazgo adecuado para obtenerlo.

Otro punto crítico de los artículos que se revisan en este trabajo, es que no han considerado autores ni investigaciones de los últimos dos años, es en esta época donde las empresas se están afectando más por la globalización y la fuerza del cambio, que ya no es solo las organizaciones sino también las personas que buscan cada día encajar en la sociedad hasta el punto de cambiar sus hábitos o costumbres solo por tener un puesto en la famosa sociedad moderna.

En la actualidad está la verdadera respuesta de cómo enfrentarse a los desafíos de una sociedad que camina hacia el futuro. Estudiar las teorías de los años 40, 70 u 80 son ayudas que permiten conocer cómo se trabajaba en esa época y como se pensaba, pero no se puede caer en un retroceso organizacional.

Las leyes de esta época, las conquistas laborales, la naturaleza del negocio y las nuevas demandas tecnológicas hacen que las empresas busquen el cambio. Los autores modernos tienen otro factor que investigar que es la sociedad como motor de cambio, ya no las industrias que cambian a la sociedad. Hoy se está en la era en que las empresas tienen que satisfacer las necesidades de los clientes que cada día son más exigentes, y las organizaciones deben saber empoderar a los trabajadores.

\section{Conclusiones}

El artículo de Cristian Benavente (2011), muestra la competitividad necesaria para la empresa moderna, administraciones y a las organizaciones con sus gerentes. Es necesario que un gerente o jefe defina anticipadamente la necesidad del cambio dentro de la empresa, para preparar a la organización al cambio. Así la probabilidad del éxito del control y transformación del cambio es mayor.

Para generar un cambio se requiere una meta y una ruta clara para alcanzar el objetivo. Una clara orientación de los empleados de la empresa es indispensable, ya que, sin la aceptación de los ellos para el cambio, puede implicar un factor negativo que muchas veces es causa de fracaso, 
como consecuencia de las resistencias de parte de los trabajadores. Un cambio, no es un proceso único ni a corto plazo. Un cambio resulta siembre en otro cambio.

Para generar mejores resultados los empleados tienen que entender que deben actuar con responsabilidad propia frente a los gerentes o jefes. Cada persona tiene que decidir por sí mismo como quiere y puede desarrollar la nueva situación en que la organización se encuentra, por eso la directiva tiene la obligación de dar la información completa a los empleados.

Los directivos tienen que tener la meta de construir un clima, donde se pueda discutir en forma constructiva y directa las equivocaciones, para aprender de ellos. La idea es aprender de estos errores y no esconderlos, porque éstos dan una oportunidad para una organización aprendida. La meta del Change Management es como dice Benavente (2011) una transformación con la gente y no contra la gente.

Es preciso la interacción entre los trabajadores y los jefes, es decir tener los canales de comunicación claros y específicos donde el empleado cuente con todos los puntos claros y no dejar nada a supuestos.

\section{Referencias bibliográficas}

Avolio, B., Bass, B., \& Jung, D. (1999). Re-examining the components of transformational and transactional leadership using the Multifactor Leadership Questionnaire. Journal of Occupational and Organizational Psychology, 441-462.

Bass, B., \& Avolio, B. (1990). Transformational Leadership development. Palo Alto: Consulting Psychologist PRess Inc.

Kraus, G., Becker-Kolle, C., \& Fischer, T. (2004). Handbuch Change Management. Berlin.

Maldonado, I., \& Pérez, M. (2006). Clima organizacional y gerencia: inductores del cambio organizacional. Investigación y Postgrado 21 (2), 231-248. 
Oria, G. (2002). Los gerentes públicos en la gestión del cambio. VII Congreso Internacional del CLAD sobre la REforma del Estasdo y de la administración pública. Lisboa.

Robbins, S. (2004). Comportamiento organizacional. México: Pearson Education.

Vargas, H. (2000). Impacto de las tendencias políticas y sociales en la formación. Gestión 2000. 